[0212-7199 (2006) 23: 2; pp 83-85] ANALES DE MEDICINA INTERNA Copyright (C) 2006 ARAN EDICIONES, S.L.

AN MED INTERNA (Madrid) Vol. 23, N. ${ }^{\circ} 2$, pp. $83-85,2006$

\title{
Un caso de carcinoma de trompa de Falopio
}

\author{
F. MARCOS SÁNCHEZ, E. SÁNCHEZ DÍAZ1, D. MARRUPE GONZÁLEZ², \\ M. I. ALBO CASTAÑO, A. VIANA ALONSO2 ${ }^{2}$, F. JUÁREZ UCELAY ${ }^{2}$ \\ Servicios de Medicina Interna, ${ }^{1}$ Anatomía Patológica y ${ }^{2}$ Oncología. Hospital Nuestra \\ Señora del Prado. Talavera de la Reina. Toledo
}

CARCINOMA OF THE FALLOPIAN TUBE: A CASE

\section{RESUMEN}

Presentamos el caso de una mujer de 43 años de edad, con antecedentes personales de retraso mental y síndrome depresivo mayor, que consultó por metrorragias. A la exploración física se palpaba una masa anexial derecha. En la ecografía transvaginal se observó un quiste en el ovario derecho con 3 cavidades.

Se decidió realizar intervención quirúrgica, efectuándose una histerectomía total con doble anexectomía.

El resultado de la anatomía patológica fue de endometrio proliferativo, quistes foliculares bilaterales y adenocarcinoma seroso bien deferenciado de la trompa de Falopio.

La paciente rechazó la realización de tratamiento con quimioterapia adyuvante y se está efectuando un seguimiento estrecho de la misma en las consultas de Oncología.

Posteriormente comentamos algunos aspectos epidemiológicos, etiológicos, clínicos, diagnósticos y terapéuticos de esta rara tumoración.

PALABRAS CLAVE: Carcinoma de trompa de Falopio. Metrorragia. Agresividad.

\begin{abstract}
The case of a 43-years old woman with previous history of mental retardation and major depressive syndrome, consulting for metrorrhagia is presented.

At physical examination a right adnexal mass was detected on palpation. Transvaginal echography showed a 3-cavity cyst in right ovary.

A surgical intervention with total hysterectomy and dual adnexectomy was performed. Pathological study showed proliferative endometrium, bilateral follicular cysts and well differentiated serous adenocarcinoma located at fallopian tube.

The patient refused adyuvant chemotherapy, a close follow-up is ongoing in outpatient Oncology Unit.

Then some epidemiologic, etiologic, clinica, diagnostic and therapeutic issues related to this rare tumour are discussed.
\end{abstract}

KEY WORDS: Fallopian tube carcinoma. Metrorrhagia. Agresivity.

Marcos Sánchez, F, Sánchez Díaz E, Marrupe González, D, Albo Castaño MI, Viana Alonso A, Juárez Ucelay F. Un caso de carcinoma de trompa de falopio. An Med Interna (Madrid) 2006; 23: 83-85.

\section{INTRODUCCIÓN}

Los tumores malignos primitivos de la trompa de Falopio son los tumores menos frecuentes del aparato genital, constituyendo del 0,1 al $0,5 \%$ del total. El pico de mayor incidencia ocurre alrededor de los 60 años de edad.

Se refiere una estrecha asociación con las inflamaciones crónicas de la trompa, sobre todo las de etiología tuberculosa, aunque también de otro origen, pero es evidente que los procesos inflamatorios tubáricos suelen ser frecuentes y bilaterales y sin embargo, el cáncer de trompa es muy infrecuente y unilateral (1-3).
Deben aplicarse unos criterios muy rigurosos para considerar una lesión neoplásica originaria de éste órgano, dado que la proximidad de la mucosa endometrial y del ovario suelen dificultar enormemente el diagnóstico cuando el tumor tiene un gran tamaño y existe afectación simultánea (3).

Dado su rareza, la mayoría de los casos comunicados ha sido series cortas, salvo algún estudio publicado en los últimos años y que comprendía más de 100 enfermas (4).

Recientemente hemos tenido ocasión de diagnosticar un nuevo caso y nos parece interesante comunicarlo por su rareza. 


\section{CASO APORTADO}

Mujer de 43 años de edad, con antecedentes personales de retraso mental, síndrome depresivo mayor, que incluso había precisado ingreso en un centro psiquiátrico, en tratamiento actualmente con clomipramina, levomepromazina, clorazepato dipotásico y lamotrigina, e hipotiroidismo en tratamiento con levotiroxina.

Había tenido 5 hijos sanos, la última menstruación había sido tres semanas antes.

Consultó por un cuadro de metrorragias. No dolor abdominal ni síndrome constitucional. A la exploración ginecológica mostró unos genitales externos de multípara, vagina normal, cuello bien epitelizado, se observó un sangrado de cavidad en abundante cantidad, útero normal y una masa anexial derecha difícil de delimitar. La exploración cardiaca y pulmonar fueron normales.

Se realizó una ecografía transvaginal con el hallazgo de un quiste en el ovario derecho de 113 x $90 \mathrm{~mm}$, con tres cavidades, una de 87 x $60 \mathrm{~mm}$, otra de $36 \times 20 \mathrm{~mm}$ y la tercera de $26 \times 15 \mathrm{~mm}$, El ovario izquierdo no se visualizó.

Analíticamente, el hemograma mostró 7500 leucocitos con 4000 neutrófilos, hemoglobina de 11 gramos/dl y 265.000 plaquetas, la bioquímica fue normal. Antígeno carcinoembrionario de $0,93 \mathrm{ng} / \mathrm{ml}$, alfafetoproteína de $1,9 \mathrm{ng} / \mathrm{ml}$ y Ca 125 de 34,6 U/ml, estando los tres marcadores en los rangos de la normalidad. Radiografía de tórax y ECG normales.

La paciente fue intervenida quirúrgicamente, efectuándose una histerectomía total con doble anexectomía.

El resultado de la anatomía patológica fue de útero con endometrio proliferativo, miometrio sin lesiones histológicas relevantes, quistes foliculares ováricos bilaterales y adenocarcinoma seroso bien diferenciado de trompa de Falopio, existiendo afectación de la serosa. Con tinción de hematoxilina-eosina se observa que la luz presenta numerosas papilas revestidas por un epitelio atípico y que existe infiltración de la serosa (Fig. 1). En la figura 2 se observan fibras musculares inmunorreactivas para desmina en las pared.

Durante su ingreso precisó atención psiquiátrica, finalmente se procedió al alta a los 14 días del ingreso.

Posteriormente fue remitida a la consulta de Oncología. La paciente rechazó cualquier otro tipo de tratamiento complementario, por lo que actualmente se está efectuando un seguimiento estrecho de la misma en consultas externas.

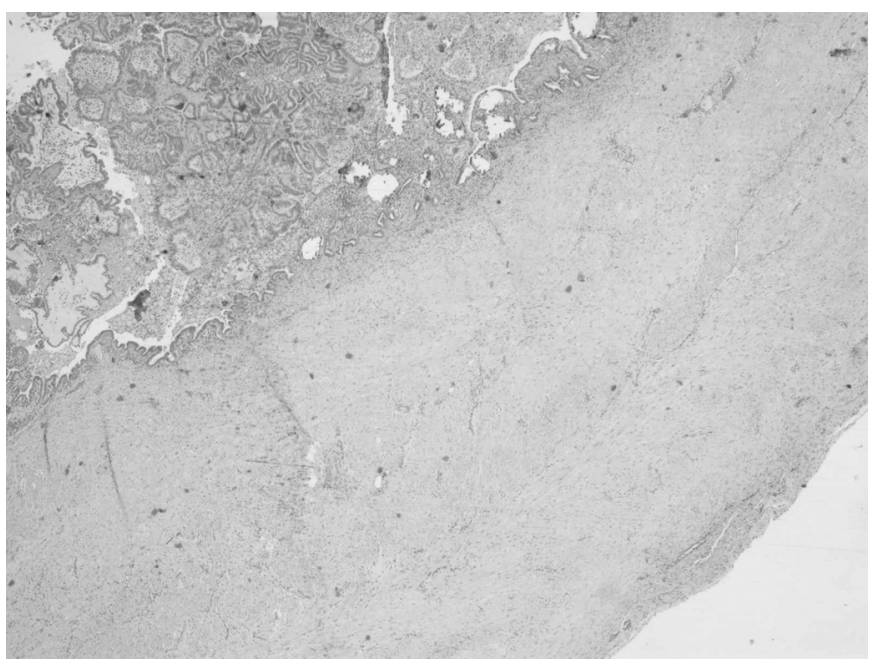

Fig. 1. La luz presenta numerosas papilas revestidas por epitelio atípico con infiltración de la serosa (hematoxilina-eosina).

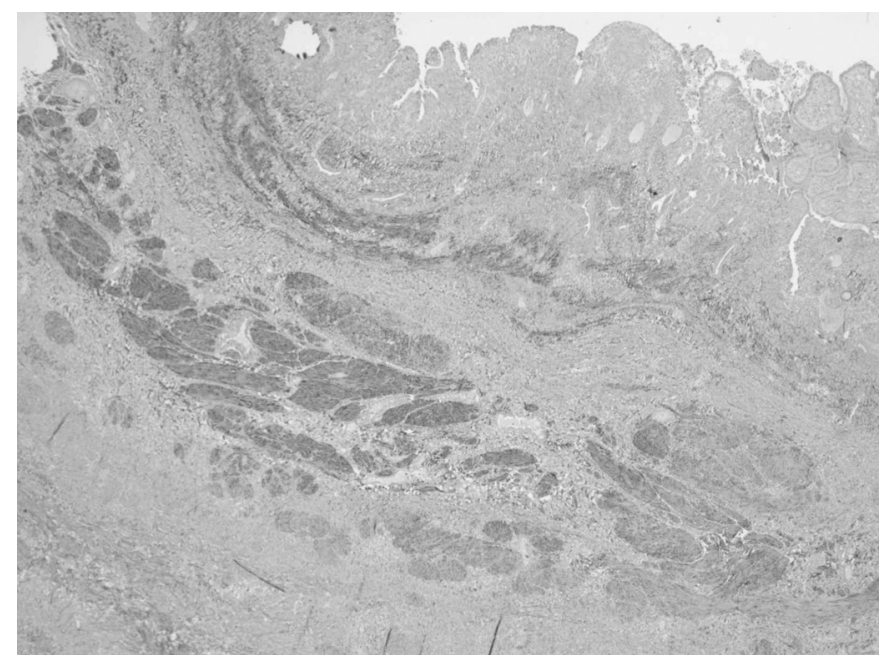

Fig. 2. Fibras musculares inmunorreactivas para desmina en la pared.

\section{DISCUSIÓN}

En el carcinoma de trompa de Falopio no se han encontrado factores de riesgo hormonal. Los factores genéticos pueden tener un papel importante, así se piensa que puede asociarse a mutaciones de los genes BRCA-1 y BRCA-2 (3).

El signo clínico más frecuente es el sangrado vaginal, en ocasiones puede existir dolor abdominal bajo, en los casos de torsiones o si hay obstrucción tubárica, menos frecuentemente se producirá distensión abdominal o ascitis en los casos de carcinomatosis peritoneal. En la mayoría de los casos el diagnóstico se produce después de realizar una laparotomía exploradora por la existencia de una masa anexial. El diagnóstico preoperatorio sólo se realiza en el $3 \%$ de los casos (3).

La ecografía en tiempo real, la ecografía tridimensional y la tecnología doppler color transvaginal han permitido realizar diagnósticos de sospecha. Así el hallazgo ecográfico más habitual es el de una masa de ecogenicidad heterogénea y flujo de baja resistencia, independiente del útero y con visualización del ovario homolateral de aspecto normal (3).

En el diagnóstico diferencial se incluyen el hematosálpinx, la enfermedad inflamatoria pélviana y el embarazo ectópico $(3,5)$.

La TAC y la RNM aportan información sobre la masa, sus relaciones anatómicas y sobre la posible existencia de ganglios paraaórticos y pélvicos. La laparoscopia debe realizarse en casos de sospecha en pacientes postmenopáusicas.

En ocasiones existe elevación de los niveles séricos de CA 12.5 y CA 19.9 (3).

Los cánceres metastásicos en la trompa se originan principalmente en el ovario y en el endometrio y son mucho más frecuentes que el carcinoma primitivo, que siempre se trata de un adenocarcinoma, siendo la variedad más frecuente la serosa. Cuando existe diseminación peritoneal, el comportamiento será similar al del cáncer de ovario; la diseminación por vía hemática es menos frecuente, con posibilidad de metástasis en el pulmón, hígado, hueso, etc. (3).

Se ha adaptado a la trompa la clasificación propuesta por la Federación Internacional de Ginecología y Obstetricia (FIGO) para el estadiaje del carcinoma de ovario, que posteriormente sufrió algunas modificaciones. Así en nuestro caso 
en el que existía una afectación de la serosa, se encuadraría en el estadio IC.

El tratamiento de elección es el quirúrgico y requerirá unas pautas similares a las que se realizan en el carcinoma de ovario, por lo que debe efectuar laparotomía supra e infraumbilical, citología de líquido ascítico si lo hay, lavados pélvicos y paracólicos, histerectomía total con doble anexectomía, omentectomía, apendicectomía, linfadenectomía iliopélvica bilateral y paraaórtica, biopsias selectivas del peritoneo parietal y mesenterio y citología de cúpulas diafragmáticas (3).

Cuando la extensión de la enfermedad no permita la práctica de una cirugía completa, se debe practicar una cirugía citorreductora, intentando dejar la mínima cantidad de enfermedad residual, pues como sucede en el carcinoma de ovario, el volumen de la enfermedad residual influye en el pronóstico; la linfadenectomía radical también puede prolongar la supervivencia (6).

\section{Bibliografía}

1. Rose PG, Piver MS, Tsukada Y. Fallopian Tube Cancer. The Roswald Park experience. Cancer 1990; 6: 2661-2667.

2. Rosenblatt KA, Weiss NS, Schwartz SM. Incidence of malignant fallopian tube tumors. Gynecol Oncol 1989; 35: 236-239.

3. Huidobro G, Moral E, Grande C, Casal J. Tumores malignos de la trompa de Falopio. En Guía práctica de tumores poco frecuentes de Lázaro Quintela M, Jorge Fernández M, Carrasco Álvarez J, Castellanos Díez J. Ed. You\&US, SA. Madrid 2003; 290-296.

4. Nordin AJ. Primary carcinoma of the fallopian tube: a 20 year literature review. Obstet Gynecol Survey 1994; 49: 349-361.

5. Kurjak A, Kupesic S, Jacobs I. Preoperative diagnosis of the fallopian
Se considera que los tumores de la trompa de Falopio son más agresivos en comparación que los mismos estadios en el carcinoma de ovario, por lo que la tendencia más aceptada es considerar la necesidad de tratamiento complementario en los estadios IC y superiores.

La radioterapia pélvica, abdominal y metabólica tiene un importante papel (7); también la quimioterapia sistémica con tres a seis ciclos, con esquemas basados en el platino con paclitaxel o ciclofosfamida (8).

La supervivencia tras realizar tratamiento complementario en estadios I-II, se encuentra sobre el $50 \%$ a los cinco años.

Las revisiones deben realizarse trimestralmente durante el primer año y posteriormente cada 6 meses durante al menos 4 años más. Debe efectuarse exploración física, pruebas radiológicas, como la ecografía, TAC, radiología de tórax y marcadores tumorales, fundamentalmente CEA, CA 12.5 y CA 19.9 (3).

tube carcinoma by threedimensional static and power doppler sonography. Ultrasound Obstet Gynecol 2000; 15: 246-251.

6. Di Re E, Grosso G, Raspagliesi F, Baiocchi G. Fallopian tube cancer: incidence and role of linphatic spread. Gynecol Oncol 1996; 62: 199-202.

7. Schray MF, Podratz KC, Malkasian GD. Fallopian tube cancer: the role of radiation therapy. Radiother Oncol 1987; 10: 267-275.

8. Ozols RF, Schwartz PE, Eifel PJ. Cáncer de ovario, carcinoma de trompa de falopio y carcinoma de peritoneo. Cáncer de la trompa de Falopio. En Cáncer: principios y práctica de Oncología de De Vita VT, Hellman S, Rosenberg SA. Madrid: Editorial médica Panamericana-Arán, 2000. p. 1534-1540. 